\title{
Critical exponents of block-block mutual information in one-dimensional infinite lattice systems
}

\author{
Yan-Wei Dai, ${ }^{1}$ Xi-Hao Chen, ${ }^{2}$ Sam Young Cho, $, 1,3$, 因 and Huan-Qiang Zhou ${ }^{1,3}$ \\ ${ }^{1}$ Centre for Modern Physics, Chongqing University, Chongqing 400044, China \\ 2 Research Institute for New Materials and Technology, \\ Chongqing University of Arts and Sciences, Chongqing 402160, China \\ ${ }^{3}$ Department of Physics, Chongqing University, Chongqing 400044, China
}

\begin{abstract}
We study the mutual information between two lattice-blocks in terms of von Neumann entropies for one-dimensional infinite lattice systems. Quantum $q$-state Potts model and transverse field spin-1/2 XY model are considered numerically by using the infinite matrix product state (iMPS) approach. As a system parameter varies, block-block mutual informations exhibit a singular behavior that enables to identify critical points for quantum phase transition. As happens with the von Neumann entanglement entropy of a single block, at the critical points, the block-block mutual information between the two lattice-blocks of $\ell$ contiguous sites equally partitioned in a latticeblock of $2 \ell$ contiguous sites shows a logarithmic leading behavior, which yields the central charge $c$ of the underlying conformal field theory. As the separation between the two lattice-blocks increases, the mutual information reveals a consistent power-law decaying behavior for various truncation dimensions and lattice-block sizes. The critical exponent of block-block mutual information in the thermodynamic limit is estimated by extrapolating the exponents of power-law decaying regions for finite truncation dimensions. For a given lattice-block size $\ell$, the critical exponents for the same universality classes seem to have very close values each other. Whereas the critical exponents have different values to a degree of distinction for different universality classes. As the lattice-block size becomes bigger, the critical exponent becomes smaller.
\end{abstract}

PACS numbers:

\section{INTRODUCTION}

As an information of one system about another, correlations quantify a relationship or connection between them. Correlations have long been a central theme of physical quantities in characterizing a unique property of strongly correlated systems in condensed matters. Conventional two-point spatial correlation functions have been studied and their scaling behaviors have been then used to characterize quantum phases of many-body systems [1, 2]. Recently much attentions have been drawn to quantum entanglement that can quantify unique correlations present in quantum states. Such as entanglement entropy, concurrence, and Rényi entropy, quantum information theoretical tools have been shown to be useful to investigate quantum critical points and different phases in strongly correlated systems [3].

Similar to conventional two-point correlations, in general, a correlation between two blocks embedded in a large system may also be considered to study a characteristic behavior of the system. For a chosen size of blocks, block-block correlations can be in principle from either classical or quantum origin. Not due to entanglement, nontrivial quantum correlations can exist [4]. Although correlations can be induced from such different origins and specific dominant correlations are not known to characterize the system, the mutual information can

\footnotetext{
*E-mail:sycho@cqu.edu.cn
}

be used to measure all kinds of correlations of one block about the other, i.e., the total amount of classical and quantum correlations between two blocks [5 8]. In terms of the von Neumann entropies, the mutual information $I(A: B)$ between two lattice-blocks $A$ and $B$ (see Fig. 11) can be defined as

$$
I(A: B)=S(A)+S(B)-S(A \cup B),
$$

where $S(\alpha)=-\operatorname{Tr} \rho_{\alpha} \log _{2} \rho_{\alpha}$ is the von Neumann entropy for the lattice-blocks with $\alpha \in\{A, B, A \cup B\}$. To calculate the mutual information between two lattice-blocks in the system, the density matrix $\rho_{\alpha}$ can be expressed in terms of expectation values of operators in the blocks. The elements of the density matrix have the form of generalized correlations functions and contain, by definition, all block-site correlations. The block-block mutual information in terms of the von Neumann entropy consists of a weighted average of generalized correlation functions and, in fact, measures the strength of the overall correlation between two blocks of sizes $\ell_{A}$ and $\ell_{B}$. This implies that without knowing a dominant correlation between blocks in the system and its corresponding operator, the mutual information can capture a characteristic property of the system even if hidden or exotic correlations present. The two-point pairwise mutual information has then been used to study quantum phase transitions [1122, [58]. A recent study shows that similar to two-point spatial correlations, two-point pairwise mutual information can characterize one-dimensional quantum critical systems by using its critical exponent [58]. In contrast with two-point pairwise mutual information, criticality of 
(a)
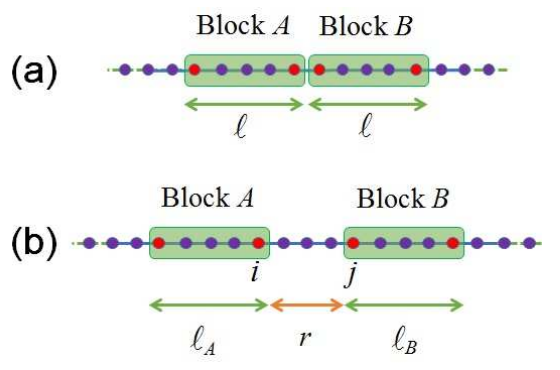

FIG. 1: (color online) Two (a) adjacent and (b) disjoint lattice-blocks $A$ and $B$ in one-dimensional infinite lattice systems. The block sizes are denoted by $l_{A}$ and $l_{B}$, respectively. The size of the blocks $l_{A}$ and $l_{B}$ are denoted by the number of sites inside the blocks, respectively. $r=|i-j|$ is the lattice distance between two blocks.

systems has also been studied by considering the mutual information between the two lattice-blocks for bipartite systems 19 22].

In order to more deeply understand mutual information in critical systems, it would be interesting to study mutual information between lattice-blocks embedded in infinite lattice systems in Fig. 1. Two consecutive subchains in one-dimensional spin models (e.g., see Fig. 1 (a)) exhibit a logarithmical growth of the entanglement with respect to the size of subchains at critical points. Compared with such two consecutive subchains, no correlation between disjoint lattice-blocks (e.g., see Fig. 1(b)) is expected for a large separation at a transition. Then it would be interesting to study (i) how this correlation between disjoint lattice-blocks behaves with their separation $r$, i.e., how it scales, at a quantum phase transition and also (ii) how the size $\ell$ of the lattice-blocks affects the scaling. Such blocks in critical systems [23 41] have been considered to investigate the entanglement entropy, the entanglement negativity, and the mutual information. Especially, the scaling functions of the von Neumann entanglement entropy (mutual information) have been studied for the Ising model [30, 32, 40] and for the spin1/2 XXZ model [24,40]. Actually, studying their scalings has been found to be a nontrivial task because the scaling functions depend not only on the central charge but also on more universal information of the conformal field theory [23 25].

In this paper we investigate the block-block mutual information between two lattice-blocks in infinitelattice systems by using the infinite matrix product state (iMPS) representation with the infinite time-evolving block decimation (iTEBD) method [9, 10, 42]. To consider various universality classes at critical points, we consider quantum $q$-state Potts model and transverse field spin-1/2 XY model and calculate the von Neuman mutual information for various sizes of lattice-blocks. We demonstrate that the block-block mutual information can be a useful probe for detecting quantum phase transition. The scaling of the block-block mutual information is studied at the critical point in the thermodynamic limit. The numerical results show that similar to conventional two-point correlations, block-block information exhibits power-law decaying behaviors. We find that as the size of lattice-block $\ell$ increases, the critical exponent decreases. The scaling behaviors of groundstate blockblock mutual informations are discussed in associations with a characterization of critical systems.

This paper is organized as follows. In Sec. II, we briefly introduce one-dimensional $q$-state quantum Potts model and numerical iMPS approach. A singular behavior of block-block mutual information appears to identify a quantum phase transition. As the size of lattice-block increases, block-block mutual information increases. In Sec. III, at the critical point, such increments of blockblock mutual information between two lattice-blocks of $\ell$ contiguous sites equally partitioned in a block of $2 \ell$ contiguous sites show a logarithmic scaling behavior. Its characteristic numerical scaling coefficient relying on $q$ is discussed in connection with a central charge in terms of block-block mutual information. In Sec. IV, blockblock mutual information is shown to decrease as the separation of blocks increases. Its power-law decaying behaviors and the critical exponents are discussed in the thermodynamic limit for transverse field spin-1/2 XY model as well as $q$-state quantum Potts model for various sizes of lattice-blocks. A summary and remarks of this work are given in Sec. V. In Appendix A, the detailed discussions on central charges from block entanglement entropies are made for one-dimensional quantum $q$-state Potts model. Appendix B shows the detailed scaling behaviors of block-block mutual informations for the transverse-field spin-1/2 XY model.

\section{ONE-DIMENSIONAL $q$-STATE QUANTUM POTTS MODEL AND MUTUAL INFORMATION}

We consider $q$-state quantum Potts model [43] with the nearest neighbor interaction in a transverse magnetic field $\lambda$. The Hamiltonian can be written as

$$
H=-\sum_{i=1}^{\infty} \sum_{p=1}^{q-1}\left[M_{x, p}^{i} M_{x, q-p}^{i+1}+\lambda M_{z}^{i}\right],
$$

where $M_{x, p}=\left(M_{x, 1}\right)^{p}$ and the Potts spin matrices $M_{x / z}$ are given as

$$
M_{x, 1}=\left(\begin{array}{cc}
0 & I_{q-1} \\
1 & 0
\end{array}\right) \text { and } M_{z}=\left(\begin{array}{cc}
q-1 & 0 \\
0 & I_{q-1}
\end{array}\right) .
$$

with the $(q-1) \times(q-1)$ identity matrix $I_{q-1}$. Due to the spontaneous symmetry breaking with the symmetry group $Z_{q}, q$-degenerate ground states emerge in the broken symmetry phases. It is known that a (dis-)continuous quantum phase transition occurs for $(q>4) q \leq 4$ in the one-dimensional quantum $q$-state Potts model [44 47].

In order to consider an one-dimensional infinite lattice of the system, we employ a wave function $|\psi\rangle$ of Hamiltonian in the iMPS representation. The iTEBD algorithm 

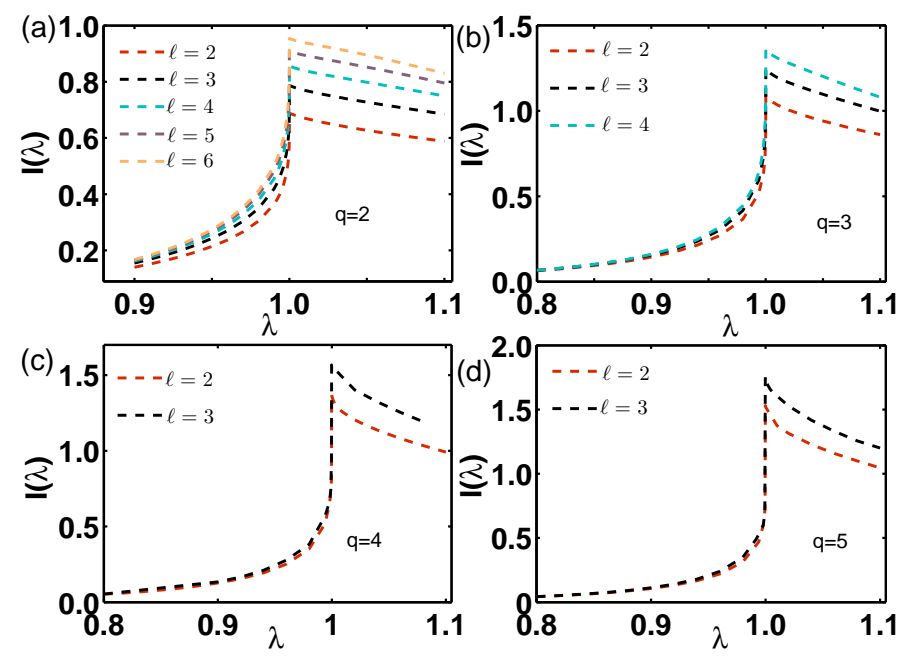

FIG. 2: (color online) Mutual information $I_{q}(\lambda)$ of two adjacent lattice-blocks as a function of the transverse field $\lambda$ for one-dimensional $q$-state quantum Potts model with various sizes of lattice-blocks $\ell$.

with the second-order Trotter decomposition leads to a numerical ground state $\left|\psi_{g}\right\rangle$ in the iMPS representation. As the initially chosen state approaches to a groundstate, according to a power law, the time step is decreased from an initial time step $d t=0.1$ to $d t=10^{-6}$. Then numerical iMPS wavefunctions for ground states are obtained for the truncation dimensions between $\chi=20$ and $\chi=150$. Actually, in the broken-symmetry phases, randomly chosen several initial states can reach different orthogonal groundstates that are degenerate ground states for a spontaneous symmetry breaking and can be distinguished by using quantum fidelity [47, 48]. Our iMPS approach gives the full description of the groundstate in a pure state by the iMPS groundstate wave function $\left|\psi_{g}\right\rangle$. The reduced density matrices $\rho_{A / B}$ are obtained from the full density matrix $\rho=\left|\psi_{g}\right\rangle\left\langle\psi_{g}\right|$ by tracing out the degrees of freedom of the rest of the lattice-blocks $A$ or $B$, i.e., $\rho_{A / B}=\operatorname{Tr}_{A^{c} / B^{c}} \rho$. Thus also $\rho_{A \cup B}=\operatorname{Tr}_{(A \cup B)^{c}} \rho$.

Based on our iMPS groundstate wavefunctions, we first consider the mutual information $I_{q}(A: B)$ between the two lattice-blocks of $\ell$ contiguous sites equally partitioned in a lattice-block of $2 \ell$ contiguous sites, i.e., $\ell_{A}=\ell_{B}=\ell$ in Fig. 1 (a). For the broken symmetry phases, i.e., $\lambda<\lambda_{c}$, if one chooses a random state as a reference state, one can detect $q$ degenerate groundstates by using the quantum fidelity [47]. All $q$ degenerate groundstates give the same block-block mutual informations. In Figs.2 (a) $q=2$, (b) $q=3$, (c) $q=4$, and (d) $q=5$, we plot the mutual informations $I_{q}(\lambda)$ as a function of the transverse field $\lambda$ for various sizes of lattice-blocks $\ell$. One can notice that compared for smaller lattice-block size, the block-block mutual information for bigger lattice-block size has a bigger value. Furthermore, all the block-block mutual informations exhibit a singular behavior for various sizes of lattice-blocks $\ell$. The singular points cor-
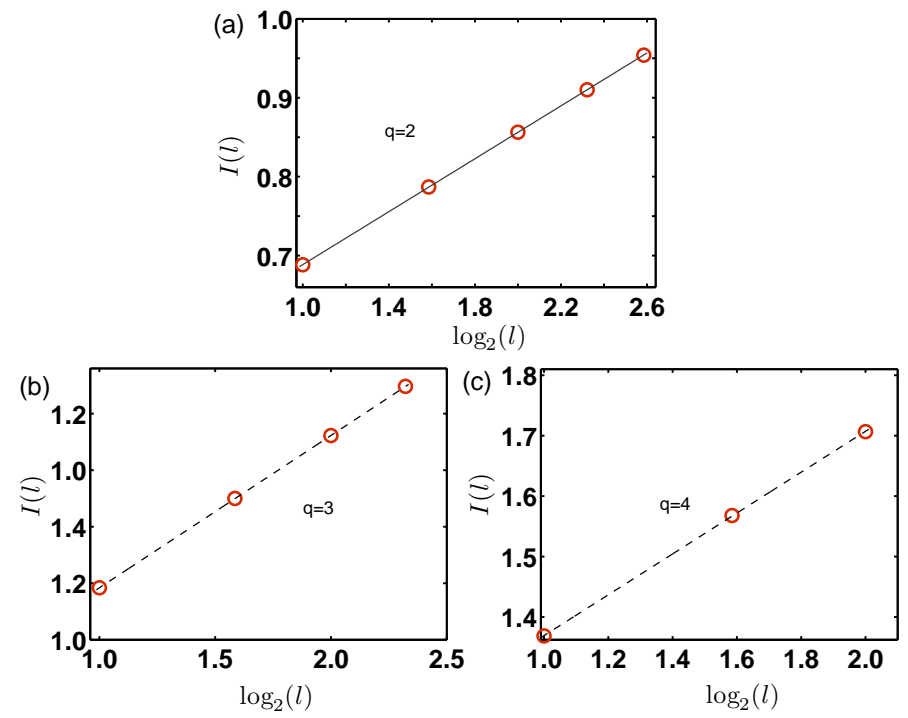

FIG. 3: (color online) Mutual information $I(\ell)$ as a function of the block length $\ell_{A}=\ell_{B}=\ell$ at the critical point $\lambda=\lambda_{c}$ for quantum Potts chains with (a) $q=2$, (b) $q=3$, and (c) $q=4$. The lines are the numerical fitting functions $I_{q}(\ell)=$ $a_{q} \log _{2} \ell+b_{q}$ with the numerical coefficients $a_{q}$ and $b_{q}$. The detailed discussions are in the text.

respond to the phase transition points $\lambda_{c}=1$. Consequently, block-block mutual information can detect quantum phase transition, which is manifested by appearing of singular behaviors.

\section{CENTRAL CHARGES AND QUANTUM MUTUAL INFORMATION}

As was discussed in the previous section, the mutual information $I_{q}(\lambda)$ between two lattice-blocks exhibits a singular behavior at the critical points, which implies that block-block mutual information can capture quantum phase transitions for spontaneous symmetry breaking. When the infinite one-dimensional chain becomes a critical system, its universality class can be identified by calculating the central charge $c$ that is the main feature of the conformal field theory for a critical system. Actually, for the ground state $\left|\psi_{g}\right\rangle$ of an infinite one-dimensional critical system and a large block of length $\ell$, conformal field theory predicts the universal scaling of the entanglement entropy [49 57] such as

$$
S(\ell)=\frac{c}{3} \log \ell+c^{\prime},
$$

where $c^{\prime}$ is a nonuniversal constant. Such a logarithmic scaling of the von Neumann entropy can be confirmed by using our iMPS approach. Our numerical estimates of central charges are in excellent agreement with the exact values at the critical points (see Appendix A).

On varying the sizes of lattice-blocks $\ell$, at the critical point, let us then consider the mutual information $I(\ell)$ 
of the system in Fig. 1 (a). We calculate the mutual information $I(\ell)$ as a function of the size of lattice-blocks $\ell$ in Fig 3. Similar to the von Neumann entanglement entropy in Eq. (4), the von Neumann mutual information $I(\ell)$ exhibits a logarithmic increment as the size of lattice-block $\ell$ increases. Also, a similar logarithmic scaling behavior of Shannon mutual information with two blocks $A$ and $B$ of sizes $\ell$ and $L-\ell$ has been conjectured for periodic chains in the ground state in Refs. [19 21], i.e., $I_{s h}(\ell, L)=c / 4 \ln [L / \pi \sin (\pi \ell / L)]+\gamma_{I}$ based on the Shanon entropy in the scaling regime $(\ell, L \gg 1)$, where $L$ is the system size and $\ell$ is the subsystem size, and $\gamma_{I}$ is the nonuniversal constant. In order to clarify the logarithmic behaviors of our mutual informations $I(A: B)$, thus we perform a numerical best fit with the fitting function

$$
I_{q}(\ell)=a_{q} \log _{2} \ell+b_{q}
$$

where $a_{q}$ and $b_{q}$ are numerical coefficients. The numerical fitting coefficients are given as (i) $a_{2}=0.1676(7)$ and $b_{2}=0.521(1)$ for $q=2$, (ii) $a_{3}=0.269(1)$ and $b_{3}=0.823(2)$ for $q=3$, and (iii) $a_{4}=0.34(2)$ and $b_{4}=1.03(3)$ for $q=4$. Actually, the coefficient of the logarithm in the entanglement entropy in Eq. (41) is dependent on the central charge $c$. For comparison with the entanglement entropy in Eq. (4), we then consider the coefficients $3 a$, i.e., (i) $3 a_{2}=0.503(2)$ for $q=2$, (ii) $3 a_{3}=0.808(4)$ for $q=3$, and (iii) $3 a_{4}=1.01(6)$ for $q=4$. One can notice that if one assumes that similar to the entanglement entropy in Eq. (4), the proportional coefficient $a_{q}$ corresponds to a central charge via $c_{q}=3 a_{q}$, our results of the central charges $c_{q}$ obtained from the mutual information $I(\ell)$ are very close to the exact results $c=0.5, c=4 / 5$ and $c=1$ for $q=2,3,4$, respectively. In Table I. the estimates of central charges $c$ obtained from the mutual information $I(A: B)$ are summarized for comparison with those values from the von Neumann block entropy (Appendix A). Consequently, it shows that the mutual information $I(\ell)$ may have a universal scaling behavior such as $I(\ell) \sim \frac{c}{3} \log \ell$ for the two lattice-blocks shown in Fig. 1 (a) in critical one-dimensional infinite lattice systems.

TABLE I: Numerical central charges $c$ estimated from the quantum mutual information (MI) and the von Neumann entanglement entropy (EE) (Appdenix A) at the critical point $\lambda=\lambda_{c}$ for $q$-state quantum Potts chains

\begin{tabular}{cccc}
\hline \hline & $q=2$ & $q=3$ & $q=4$ \\
\hline$c$ & $1 / 2$ & $4 / 5$ & 1 \\
\hline$c(\mathrm{MI})$ & $0.503(2)$ & $0.808(4)$ & $1.01(6)$ \\
\hline$c(\mathrm{EE})$ & $0.5007(3)$ & $0.800(2)$ & $1.00(4)$ \\
\hline \hline
\end{tabular}

\section{SCALING BEHAVIOR OF BLOCK-BLOCK MUTUAL INFORMATION}

For strongly correlated systems, a characteristic scaling behavior of conventional (two-point) spatial correlation can quantify their property. Similarly, it has been proposed to use the mutual information which quantifies the total amount of correlations including classical and quantum correlations shared between two parties. A scaling behavior of two-point (site) spatial mutual information has been studied and discussed its universality 58] in one-dimensional critical systems. In this section we study spatial behaviors of the mutual information $I(A: B)$ between the two lattice-blocks with $\ell_{A}=\ell_{B}=\ell$ at the critical point. The effects of the lattice-block size on the behaviors of mutual information will be investigated. We will then consider various sizes of latticeblocks in investigating scaling behaviors of block-block mutual information. The detailed behaviors of mutual information will be discussed for quantum $q$-state Potts model in Subsection IVA. For the transverse-field spin1/2 XY model, we present a summary of scaling of mutual information in Subsection IVB and the detailed discussion in Appendix B.

\section{A. Block-block mutual information critical exponent $\eta^{I}$ for quantum $q$-state Potts model}

Let us first consider quantum $q$-state Potts model. As shown in Fig. 1 (b), when the two lattice-blocks are separated from each other as the distance $r$, we discuss mutual information between the two lattice-blocks. For $q=2$ (Ising chain), we plot the mutual information $I(r)$ as a function of the lattice distance $r$ in the left of Fig. 4. For given truncation dimensions, the plots show that the mutual information decreases as the lattice distance $r$ increases. With bigger truncation dimension, the linear region of the log-log plot becomes longer and the slope of the linear region seems to be readily saturated for the truncation dimension $\chi=150$ in the left of Fig. 4. This tendency implies that similar to the power-law behavior of the von Neumann entropy in [30, 40], the mutual information undergoes a power-law decay to zero if the truncation dimension $\chi$ increases to the thermodynamic limit. Then the mutual information seems to decay linearly to zero, i.e., $I(A: B) \rightarrow 0$ as $r \rightarrow \infty$. This shows that for very large separation of the two lattice-blocks, $S_{A \cup B} \simeq S_{A}+S_{B}$. Such behaviors of the mutual information $I(A: B)$ can be observed for all the sizes of lattice-blocks, i.e., in Figs. 4 (a) $\ell=2$, (b) $\ell=3$ and (c) $\ell=4$.

To confirm the power-law decay of mutual information $I(r)$, we perform a numerical fit for the linear region of mutual information $I(r)$ with the fitting function, $\log I(r)=\eta^{I} \log r+a_{0}$, where $\eta^{I}$ corresponds to an exponent of power-law decay and $a_{0}$ is a fitting constant. In the right of Fig. 4, we plot the slopes $\eta^{I}(\chi)$ of the 

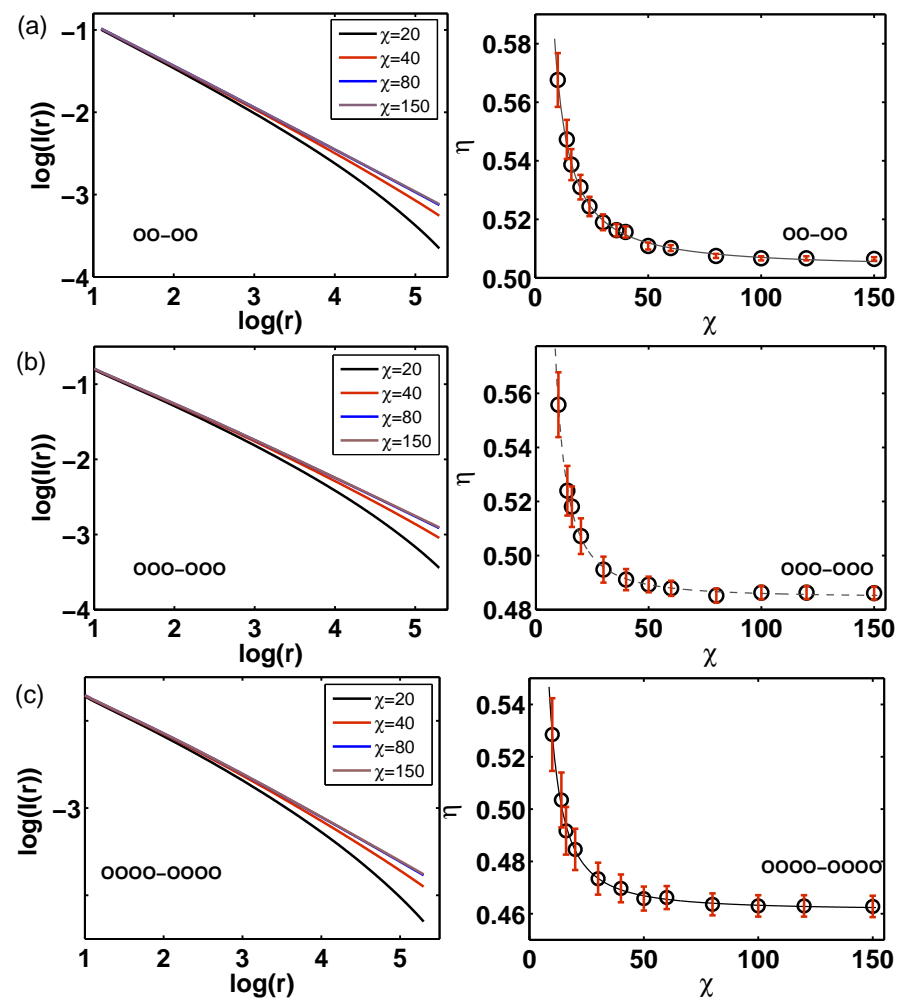

FIG. 4: (color online) Mutual information $I(r)$ as a function of the lattice distance $r=|i-j|$ for various truncation dimensions (left) and mutual information exponent $\eta^{I}(\chi)$ as a function of truncation dimension $\chi$ (right) with the block lengths $\ell_{A}=\ell_{B}=\ell$, i.e., (a) $\ell=2$, (b) $\ell=3$, and (c) $\ell=4$ for Ising chain $(q=2)$. Mutual information exponent $\eta^{I}(\chi)$ (right) is extracted from the numerical fitting of the mutual information $I(\chi)$ (left) with the fitting function $\log I(r)=\eta^{I} \log r+a_{0}$ for the power-law decaying part. The detailed discussions are in the text.

linear regions as a function of the truncation dimension $\chi$ with the fitting error bars. The $\eta^{I}$ decreases monotonically to a saturated value as the truncation dimension $\chi$ increases. In order to obtain the exponent $\eta_{\infty}^{I}$ of mutual information in the thermodynamic limit $\chi \rightarrow \infty$, we extrapolate the exponents for various truncation dimension in the right of Fig. 4 The extrapolation functions are employed as a form of $\eta^{I}(\chi)=\eta_{0}^{I} \chi^{\alpha}+\eta_{\infty}^{I}$. The numerical estimates of the critical exponent $\eta_{\infty}^{I}$ of mutual information in the thermodynamic limit are given as (a) $\eta_{0}^{I}=1.2(2), \alpha=-1.26(9)$ and $\eta_{\infty}^{I}=0.503(1)$ for $\ell=2$, (b) $\eta_{0}^{I}=3.5(9), \alpha=-1.7(1)$ and $\eta_{\infty}^{I}=0.485(1)$ for $\ell=3$, and (c) $\eta_{0}^{I}=2.4(8), \alpha=-1.6(1)$ and $\eta_{\infty}^{I}=0.461(2)$ for $\ell=4$. For bigger size of latticeblock, the critical exponent $\eta_{\infty}^{I}$ becomes smaller. This means that the larger the lattice-blocks are, the slower the block-block information decays to zero as the lattice distance $r$ increases. In contrast to the mutual information, the von Neumann entropy was shown to be scaled as $S_{A B} \sim r^{-1 / 2}$ independent on the block sizes in [30, 40].

For three-state Potts model $(q=3)$, in the left of
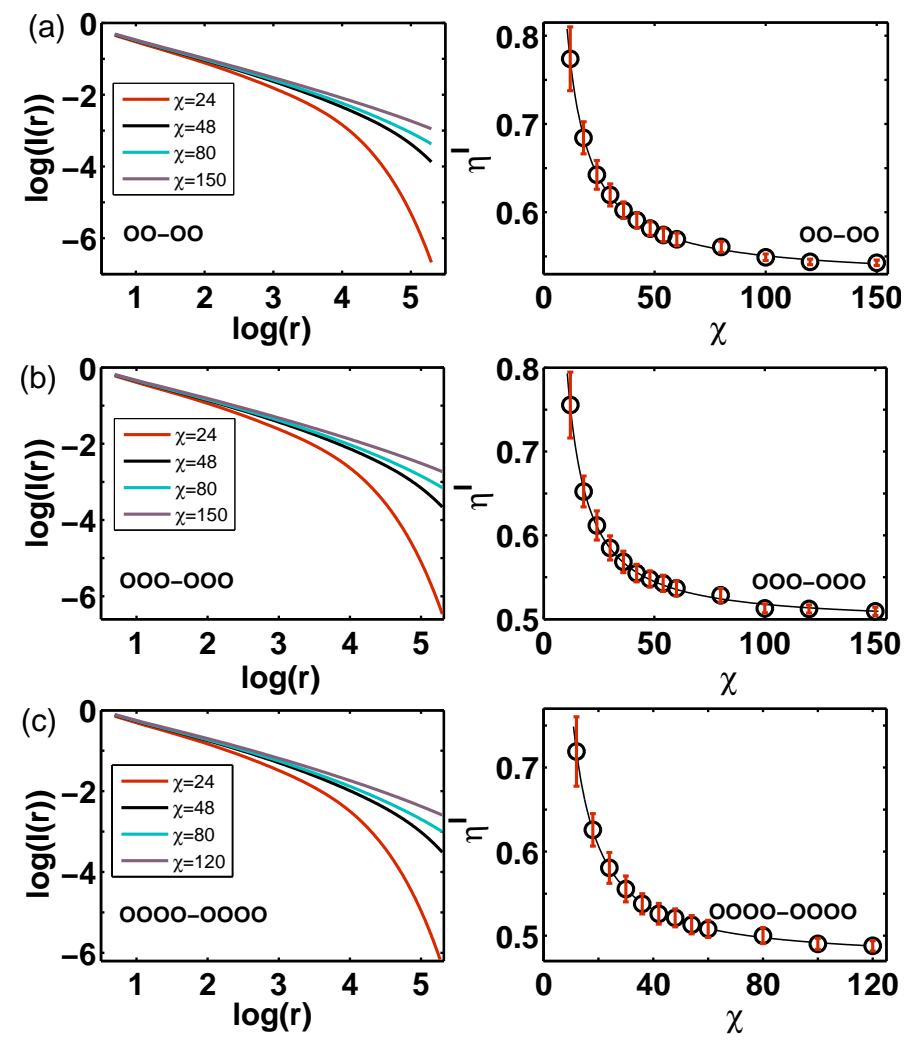

FIG. 5: (color online) Mutual information $I(r)$ as a function of the lattice distance $r=|i-j|$ for various truncation dimensions (left) and mutual information exponent $\eta^{I}(\chi)$ as a function of truncation dimension $\chi$ (right) with the block lengths $\ell_{A}=\ell_{B}=\ell$, i.e., (a) $\ell=2$, (b) $\ell=3$, and (c) $\ell=4$ for three-state Potts chain $(q=3)$. Mutual information exponent $\eta^{I}(\chi)$ (right) is extracted from the numerical fitting of the mutual information $I(\chi)$ (left) with the fitting function $\log I(r)=\eta^{I} \log r+a_{0}$ for the power-law decaying part. The detailed discussions are in the text.

Fig. 5, we display the block-block mutual information $I(r)$ as a function of the lattice distance $r$ for various truncation dimension $\chi$. In Figs. 5 (a)-(c), the sizes of lattice-blocks are chosen respectively as (a) $\ell=2$, (b) $\ell=3$, and (c) $\ell=4$. For given truncation dimensions, the plots show that the mutual information decreases monotonically as the lattice distance $r$ between the two lattice-blocks increases. Similar to the case of $q=2$, as the truncation dimension $\chi$ increases, the linear region of the plot becomes longer. For $\chi=150$ in the left of Fig. [5 the slope of the linear region seems to be almost straight in the range of the plot. Regardless of the size of lattice-block $\ell$, such similar behaviors of mutual information $I(r)$ are noticeable. To analyze a characteristic behavior of mutual information, we adapt the approach for $q=2$. Using the fitting function, $\log I(r)=\eta^{I} \log r+a_{0}$, we perform the numerical fit for the linear region of mutual information $I(r)$ in the right of Fig. 5. The slopes $\eta^{I}(\chi)$ of the linear regions are plotted as a function of the truncation dimension $\chi$ 

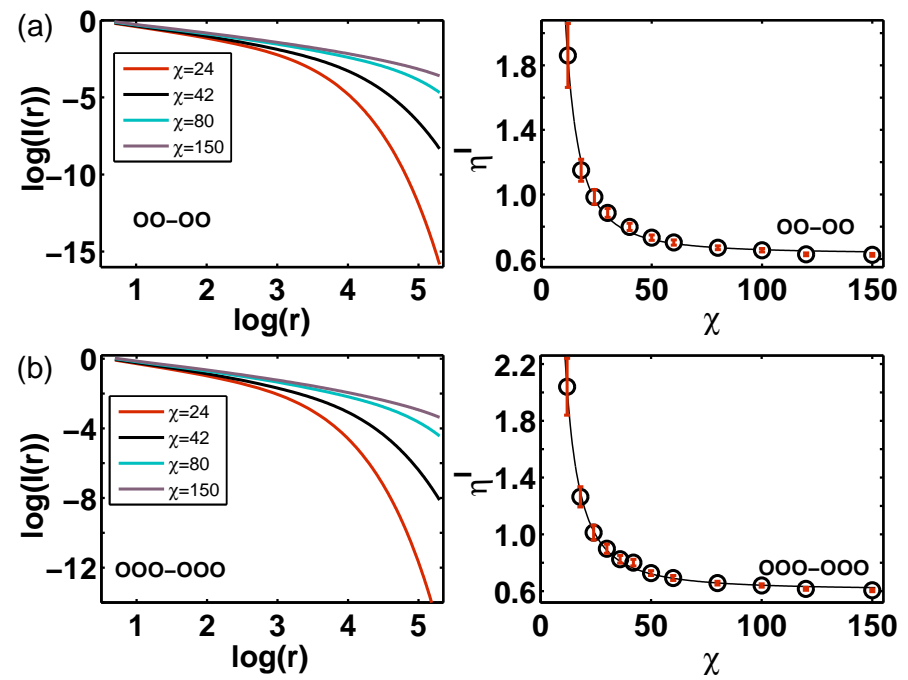

FIG. 6: (color online) Mutual information $I(r)$ as a function of the lattice distance $r=|i-j|$ for various truncation dimensions (left) and mutual information exponent $\eta^{I}(\chi)$ as a function of truncation dimension $\chi$ (right) with the block lengths $\ell_{A}=\ell_{B}=\ell$, i.e., (a) $\ell=2$ and (b) $\ell=3$ for four-state Potts chain $(q=4)$. Mutual information exponent $\eta^{I}(\chi)$ (right) is extracted from the numerical fitting of the mutual information $I(\chi)$ (left) with the fitting function $\log I(r)=\eta^{I} \log r+a_{0}$ for the power-law decaying part. The detailed discussions are in the text.

with the fitting error bars, which shows the monotonic decrement of $\eta^{I}$ with the increment of truncation dimension $\chi$. The extrapolation of the exponents is performed with the function, $\eta^{I}(\chi)=\eta_{0}^{I} \chi^{\alpha}+\eta_{\infty}^{I}$. We estimate the critical exponent $\eta_{\infty}^{I}$ of mutual information in the thermodynamic limit as (a) $\eta_{0}^{I}=3.5(4), \alpha=-1.07(5)$ and $\eta_{\infty}^{I}=0.525(4)$ for $\ell=2$, (b) $\eta_{0}^{I}=4.7(9), \alpha=-1.16(8)$ and $\eta_{\infty}^{I}=0.496(6)$ for $\ell=3$, and (c) $\eta_{0}^{I}=4.7(5)$, $\alpha=-1.19(5)$ and $\eta_{\infty}^{I}=0.472(3)$ for $\ell=4$. For $q=3$, the estimates of the critical exponent $\eta_{\infty}^{I}(\ell)$ show that as the size of lattice-block $\ell$ becomes bigger, the $\eta_{\infty}^{I}(\ell)$ becomes smaller. Consequently, similar to the Ising chain for $q=2$, the mutual information $I(r)$ for $q=3$ follows an asymptotic power-law scaling with a different scaling exponent.

The block-block mutual information $I(r)$ reveals a very similar behavior for $q=2$ and $q=3$. The critical exponents seem distinguishable each other for a given latticeblock size. In order to clarify the criticality, the critical point of the four-state quantum Potts model $(q=4)$ belonging to another universality class needs to be considered. Let us then consider four-state quantum Potts model $(q=4)$. We plot the mutual information $I(r)$ for the sizes of lattice-blocks (a) $\ell=2$ and (b) $\ell=3$ in Fig. 6. Similar to the cases of $q=2$ and $q=3$, the mutual information $I(r)$ for $q=4$ exhibit a power-law decaying tendency as the truncation dimension $\chi$ increases. By using the same numerical method for $q=2$ and $q=3$, we estimate the critical exponents of the mutual informa- tion $I(r)$ for the block lengths $\ell=2$ and $\ell=3$ in the right of Fig. 6. Performing the extrapolation with the fitting function $\eta^{I}(\chi)=\eta_{0}^{I} \chi^{\alpha}+\eta_{\infty}^{I}$, we get the fitting results as (a) $\eta_{0}^{I}=115(73), \alpha=-1.8(3)$ and $\eta_{\infty}^{I}=0.63(4)$ for $\ell=2$ and (b) $\eta_{0}^{I}=118(43), \alpha=-1.8(1)$ and $\eta_{\infty}^{I}=0.61(3)$ for $\ell=3$. As expected from the cases of $q=2$ and $q=3$, the exponent $\eta_{\infty}^{I}$ is smaller for $\ell=3$ than for $\ell=2$. For a given size of lattice-blocks, the exponent $\eta_{\infty}^{I}$ has a distinguishable value from those of $q=2$ and $q=3$.

Thus, for comparison, we summarize our numerical estimates of critical exponents $\eta_{\infty}^{I}$ at the critical points for one-dimensional quantum $q$-state Potts model in Table III. It is shown that for all of $q=2,3$, and 4 , the block-block mutual informations $I(r)$ undergo an asymptotic power-law scaling behavior at the critical points and the critical exponents become smaller for bigger lattice-blocks. These can be a characteristic feature of block-block mutual information for one-dimensional critical systems. Depending on $q$, i.e., universality class, the critical exponents $\eta^{I}(q, \ell)$ seem to be given in a different value each other.

\section{B. Block-block mutual information critical exponent $\eta^{I}$ for transverse field spin-1/2 XY model}

In order to clarify more about universal feature of the algebraic decay of block-block mutual information in onedimensional critical systems, we consider the transversefield spin-1/2 XY model [59 64] described by the Hamiltonian

$H_{X Y}=-\sum_{i=-\infty}^{\infty}\left[\left(\frac{1+\gamma}{2}\right) \sigma_{i}^{x} \sigma_{i+1}^{x}+\left(\frac{1-\gamma}{2}\right) \sigma_{i}^{y} \sigma_{i+1}^{y}+h \sigma_{i}^{z}\right]$

where $\sigma^{x, y, z}$ are the Pauli spin operators. This model has two parameters, i.e., the anisotropy interaction parameter $\gamma$ and the transverse magnetic field $h$. As is known, the transverse-field spin- $1 / 2 \mathrm{XY}$ model has two critical lines, i.e, (i) the Ising transition lines with the central charge $c=1 / 2$ for $\gamma \neq 0$ and $h= \pm 1$, and (ii) the anisotropy transition line with the central charge $c=1$ for $\gamma=0$ and $-1<h<1$. For $\gamma=1$, the XY model

TABLE II: Critical exponents $\eta_{\infty}^{I}(q, \ell)$ of block-block mutual information $I(A: B)$ for various lattice-block sizes $\ell_{A}=\ell_{B}=$ $\ell$ at the critical points for one-dimensional quantum $q$-state Potts model.

\begin{tabular}{c|ccc}
\hline \hline$\eta_{\infty}^{I}(q, \ell)$ & $\ell=2$ & $\ell=3$ & $\ell=4$ \\
\hline$q=2$ & $0.503(1)$ & $0.485(1)$ & $0.461(2)$ \\
\hline$q=3$ & $0.525(4)$ & $0.496(6)$ & $0.472(3)$ \\
\hline$q=4$ & $0.63(4)$ & $0.61(3)$ & \\
\hline \hline
\end{tabular}


reduces to the Ising Hamiltonian for $q=2$ in Eq. (2). Thus, in terms of the two parameters, $(\gamma, h)=(1.0,1.0)$ corresponds to the critical point of the Ising Hamiltonian. The block-block mutual information at $(\gamma, h)=(1.0,1.0)$ has been studied in Fig. (4) in Subsection IVA. For comparison with $(\gamma, h)=(1.0,1.0)$, we choose $(\gamma, h)=$ $(0.5,1.0)$ on the Ising transition line belonging to the same university class, i.e., the Ising universality class. Also another two parameter sets $(\gamma, h)=(0.0,0.0)$ and $(0.0,0.5)$ are chosen on the anisotropy transition line belonging to the Gaussian university class.

For the three parameter sets, we calculate the mutual information $I(A: B)$ with the lattice-blocks $\ell_{A}=$ $\ell_{B}=\ell=2,3$, and 4 . Similar to the one-dimensional $q$ state Potts model, the block-block mutual informations exhibit similar power-law decaying behaviors (see the details in Appendix B). In Table III their critical exponents are estimated and summarized with the case of the Ising critical point $(q=2)$. Table III shows clearly that the critical exponent $\eta_{\infty}^{I}(\gamma, h)$ decreases as the size of lattice-block $\ell$ increases. For each given lattice-block $\ell$, the two critical exponents $\eta^{I}(\gamma, h)$ of the mutual information $I(r)$ at $(\gamma, h)=(1.0,1.0)$ and $(0.5,1.0)$ display a very close value each other. The two critical exponents at $(\gamma, h)=(0.0,0.0)$ and $(0.0,0.5)$ also have a very close value each other. Moreover, it is shown that in accordance with the Ising universality class or the Gaussian universality class, the values of the critical exponents can be distinguishable each other for a given size of latticeblock.

\section{SUMMARY}

The block-block mutual information defined by the von Neumann entropies has been numerically investigated in the one-dimensional $q$-state quantum Potts model and the transverse-field spin- $1 / 2 \mathrm{XY}$ model. In order to calculate the reduced density matrices for the mutual information, the groundstate wavefunction of the infinite-size

TABLE III: Critical exponents $\eta_{\infty}^{I}(\gamma, h)$ of block-block mutual information $I(A: B)$ for various lattice-block sizes $\ell_{A}=\ell_{B}=\ell$ on the two critical lines of one-dimensional quantum transverse-field spin- $1 / 2 \mathrm{XY}$ model. For comparison, the estimates for Ising model are from Table $\llbracket$ for $q=2$.

\begin{tabular}{c|ccc}
\hline \hline$\eta_{\infty}^{I}(\gamma, h)$ & $\ell=2$ & $\ell=3$ & $\ell=4$ \\
\hline $\begin{array}{c}\mathrm{XY} \\
(\gamma=0.0, h=0.0)\end{array}$ & $0.999(4)$ & $0.970(8)$ & $0.926(5)$ \\
\hline $\begin{array}{c}\mathrm{XY} \\
(\gamma=0.0, h=0.5)\end{array}$ & $1.008(4)$ & $0.972(4)$ & $0.922(6)$ \\
\hline $\begin{array}{c}\mathrm{XY} \\
(\gamma=0.5, h=1.0)\end{array}$ & $0.501(6)$ & $0.483(3)$ & $0.452(6)$ \\
\hline $\begin{array}{c}\text { Ising }(q=2) \\
(\gamma=1.0, h=1.0)\end{array}$ & $0.503(1)$ & $0.485(1)$ & $0.461(2)$ \\
\hline \hline
\end{tabular}

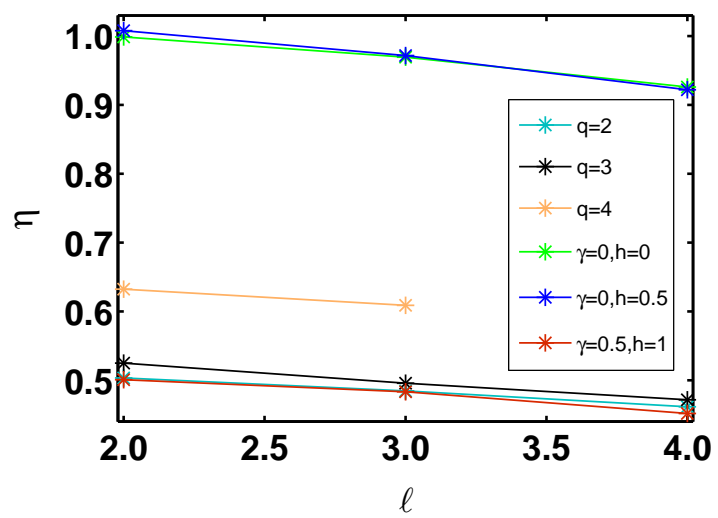

FIG. 7: (color online) Block-block mutual information exponent $\eta_{\infty}^{I}(\ell)$ as a function of the lattice-block size $\ell$ for onedimensional $q$-state quantum Potts model in Table II and the transverse-field spin-1/2 XY model in Table III

lattice chain is obtained by using the iTEBD algorithm in the iMPS representation. We first considered the mutual information $I_{q}(A: B)$ between the two blocks of $\ell$ contiguous sites equally partitioned in a block of $2 \ell$ contiguous sites, i.e., for $\ell_{A}=\ell_{B}=\ell$. For the spontaneous symmetry breaking in one-dimensional $q$-state quantum Potts model, we found that all $q$ degenerate groundstates give the same block-block mutual informations. Also, the block-block mutual informations $I_{q}(\ell)$ exhibit a singular behavior which indicates that a quantum phase transition occurs at the singular point and thus can be detected by using block-block mutual information. At the critical points, the mutual information $I_{q}(\ell)$ seems to have a logarithmic leading behavior, i.e., $I_{q}(\ell) \sim c_{q} / 3 \log \ell$ and the numerical coefficients of the logarithm are shown to be a close value of the central charge $c_{q}$ for each $q$ in Table $[$.

Next, we considered the mutual information between separated two lattice-blocks. In both the ordered and the disordered phases, as the distance $r$ between two latticeblocks increases, the block-block mutual informations exponentially decay to zero. Whereas, regardless of the size of lattice-block $\ell$, the mutual information $I(\ell)$ is shown to undergo a power-law decay at the critical points. By using the extrapolation of the exponents of $I(\ell)$ for finite truncation dimensions, the critical exponents $\eta_{\infty}^{I}$ in the thermodynamic limit were estimated at the critical points. In order to see clearly the change of critical exponent for various sizes of lattice-blocks $\ell$, we plot the critical exponent $\eta_{\infty}^{I}$ as a function of $\ell$ in Fig. 7 based on Table for the $q$-state quantum Potts model and Table III for the transverse-field spin-1/2 XY model. It is shown clearly that the larger the size of lattice-block $\ell$ becomes, the smaller the critical exponent $\eta_{\infty}^{I}$ becomes, i.e., the slower the block-block mutual information decays to zero as the distance $r$ increases. The decreasing tendencies of the mutual informations $I(\ell)$ are similar each other in all cases as the size of lattice-block $\ell$ increases. For a given lattice-block size $\ell$, the critical exponents of $I(\ell)$ for the same universality class seem to have very 

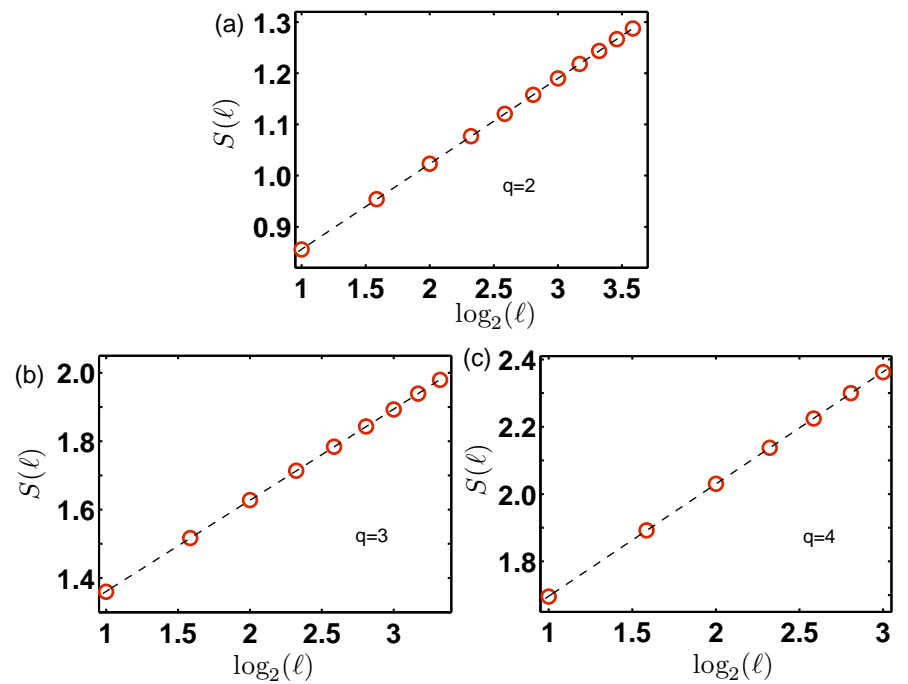

FIG. 8: (color online) Von Neumann entropy $S(\ell)$ as a function of the block length $\ell$ at the critical point $\lambda=\lambda_{c}$ for quantum Potts chains with (a) $q=2$, (b) $q=3$, and $q=4$. The lines are the numerical fitting functions $S_{q}(\ell)=f_{q} \log _{2} \ell+g_{q}$ with the numerical coefficients $f_{q}$ and $g_{q}$. The detailed discussions are in the text.

close values each other in Table III in Subsection IVB Whereas for different universality classes, the values of critical exponents are different distinguishably.

\section{Acknowledgments}

YWD is supported in part by NSFC-11805285, and the Fundamental Research Funds for the Central Universities (Grant No. 2019CDXYXDWL30030). XHC is supported by Talent Introduction Research Funds of CQWU (Grant No. R2019FXCo7). SYC acknowledges support in part from the National Natural Science Foundation of China (Grant Nos. 11674042, and 11174375).

\section{Appendix A: Central charges and block entanglement entropy}

For a bipartite system, the entanglement between the two parties is defined as the von Neumann entropy. If one of the two parties is described by the reduced density matrix $\rho_{\ell}$ for a lattice-block of $\ell$ contiguous sites, the von Neumann entropy is given as $S\left(\rho_{\ell}\right)=-\operatorname{Tr}\left[\rho_{\ell} \ln \rho_{\ell}\right]$. When a system is in a critical regime, the conventional two-point correlation length diverges and correlations remain finite even at very large length scales. For one dimensional systems, conformal field theory, describes the continuum limit for critical systems, predicts an explicit form of the von Neumann entropy which relates the coefficient of logarithmic correction to the central charge of the theory. The entanglement entropy shows a universal behavior given in Eq. (4). In this Appendix, we calculate the von Neumann entropy for various lattice-block of $\ell$ contiguous sites in our iMPS groundstates. In Fig. 8, we plot the block entanglement entropy as a function of the lattice-block size $\ell$ for the one-dimensional (a) $q=2$, (b) $q=3$, and (c) $q=4$ state Potts models. As was predicted in Eq. (44), the entanglement entropies in Fig. 8 exhibit a logarithmic scaling behavior. This fact can be manifested by performing numerical fits to extract central charges. With the fitting function $S_{q}(\ell)=f_{q} \log _{2} \ell+g_{q}$ with the numerical coefficients $f_{q}$ and $g_{q}$. The numerical fitting coefficients are given as (a) $f_{2}=0.1669(1)$ and $g_{2}=0.6892(4)$ for $q=2$, (b) $f_{3}=0.266(8)$ and $g_{3}=1.094(2)$ for $q=3$, and (c) $f_{4}=0.3334(13)$ and $g_{4}=1.363(3)$ for $q=4$. From the fitting coefficients, the central charges can be estimated as $3 f_{q}=c_{q}$, i.e., (a) $3 f_{2}=0.5007$ (3) for $q=2$, (b) $3 f_{3}=0.800(2)$ for $q=3$, and (c) $3 f_{4}=1.00(4)$ for $q=4$. Our estimates of the central charges $c_{q}$ obtained from the von Neumann entropy $S_{q}(\ell)$ are in excellent agreement with the exact values as was shown in Table II This shows that the iMPS approach gives a reliable numerical result for the central charges.

\section{Appendix B: Block-block mutual information for transverse spin-1/2 XY model}

In this Appendix, we will consider the model Hamiltonian in Eq. (6) to investigate the criticality of blockblock mutual information $I(A: B)$. In the $\gamma$ - $h$ parameter space, the four parameters are chosen for the two critical lines as (i) $(\gamma, h)=(1.0,1.0)$ and $(0.5,1.0)$ on the Ising transition line and (ii) $(\gamma, h)=(0.0,0.0)$ and $(0.0,0.5)$ on the anisotropy transition line. For the parameters $(\gamma, h)=(1.0,1.0)$ corresponding to the Ising model for $q=2$, the results of the exponents of mutual informations are displayed in Fig. 4.

Figure 9 displays the results of the mutual informations and their exponents for $(\gamma, h)=(0.5,1.0)$. Overall behaviors of the mutual informations in the left of Fig. 9 for $(\gamma, h)=(0.5,1.0)$ are shown to be similar with

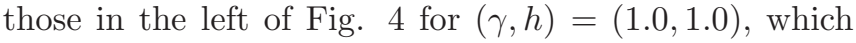
is that block-block mutual information $I(r)$ decays to zero algebraically as the distance $r$ between two blocks increases. In the right of Fig. 9, we plot the exponents $\eta(\chi)$ of block-block mutual information as a function of the truncation dimension $\chi$ for various sizes of latticeblocks $\ell$. In order to get the critical exponents $\eta_{\infty}$ in the thermodynamic limit, the extrapolations are performed with the fitting function, $\eta^{I}(\chi)=\eta_{0}^{I} \chi^{\alpha}+\eta_{\infty}^{I}$ as follows: (a) $\eta_{0}^{I}=0.21(6), \alpha=-0.6(2)$ and $\eta_{\infty}^{I}=0.501(6)$ for $\ell=2$, (b) $\eta_{0}^{I}=0.4(1), \alpha=-1.0(2)$ and $\eta_{\infty}^{I}=0.483(3)$ for $\ell=3$, and (c) $\eta_{0}^{I}=0.4(2), \alpha=-0.8(2)$ and $\eta_{\infty}^{I}=$ 0.452 (6) for $\ell=4$. One can notice that the critical exponents $\eta_{\infty}^{I}$ for $(\gamma, h)=(0.5,1.0)$ are very close values for $(\gamma, h)=(1.0,1.0)$ in Fig. 4. Note that the two parameters are in the same universality class, i.e., the Ising universality class with $c=1 / 2$. 

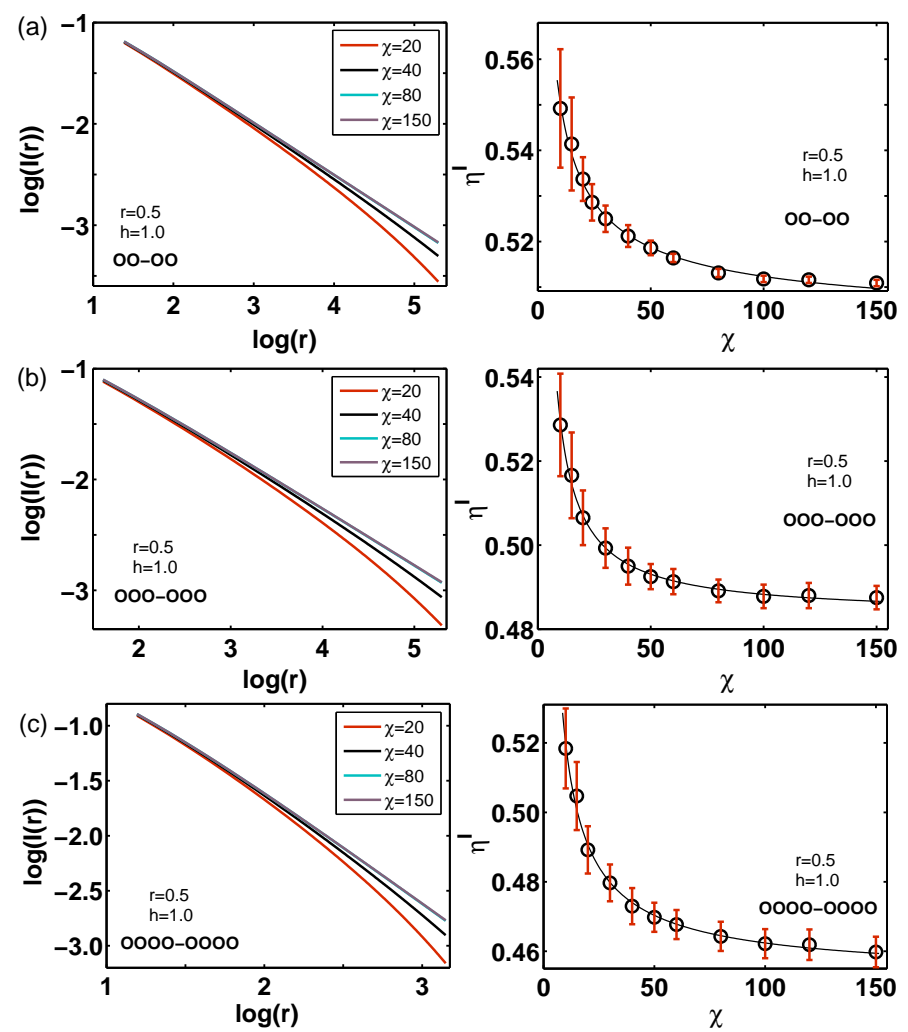

FIG. 9: (color online) Mutual information $I(r)$ as a function of the lattice distance $r=|i-j|$ for various truncation dimensions (left) and mutual information exponent $\eta^{I}(\chi)$ as a function of truncation dimension $\chi$ (right) with the block lengths $\ell_{A}=\ell_{B}=\ell$, i.e., (a) $\ell=2$, (b) $\ell=3$, and (c) $\ell=4$ for the transverse-field spin-1/2 XY model with $(\gamma, h)=(0.5,1.0)$. Mutual information exponent $\eta^{I}(\chi)$ (right) is extracted from the numerical fitting of the mutual information $I(\chi)$ (left) with the fitting function $\log I(r)=\eta^{I} \log r+a_{0}$ for the powerlaw decaying part.

Now, let us consider other two parameters $(\gamma, h)=$ $(0.0,0.0)$ and $(\gamma, h)=(0.0,0.5)$ on the anisotropy transition line belonging to the Gaussian universality class with $c=1$. In Figs 10 and 11, the results of block-block mutual informations and their exponents are displayed for $(\gamma, h)=(0.0,0.0)$ and $(\gamma, h)=(0.0,0.5)$, respectively. For the sizes of lattice-blocks (a) $\ell=2$, (b) $\ell=3$, and (c) $\ell=4$, as the distance between two blocks increases, a noticeable common behavior is an algebraic decay of block-block mutual information $I(r)$ to zero. In order to obtain the critical exponents of mutual information in the thermodynamic limit, we get the exponent $\eta^{I}(\chi)$ for a given truncation dimension $\chi$ from the fitting function $\log (I(r))=\eta^{I} \log (r)+a_{0}$. As shown in the right of Figs. 10 and 11, the extrapolations are performed for the critical exponents $\eta_{\infty}$ with the function $\eta^{I}(\chi)=\eta_{0}^{I} \chi^{\alpha}+\eta_{\infty}^{I}$. For $(\gamma, h)=(0.0,0.0)$ in Fig. 10, the numerical con- stants are given as (a) $\eta_{0}^{I}=3.3(2), \alpha=-0.93(3)$ and $\eta_{\infty}^{I}=0.999(4)$ for $\ell=2$, (b) $\eta_{0}^{I}=4.3(5), \alpha=-1.00(5)$ and $\eta_{\infty}^{I}=0.970(8)$ for $\ell=3$, and (c) $\eta_{n}^{I}=5.4(4)$,
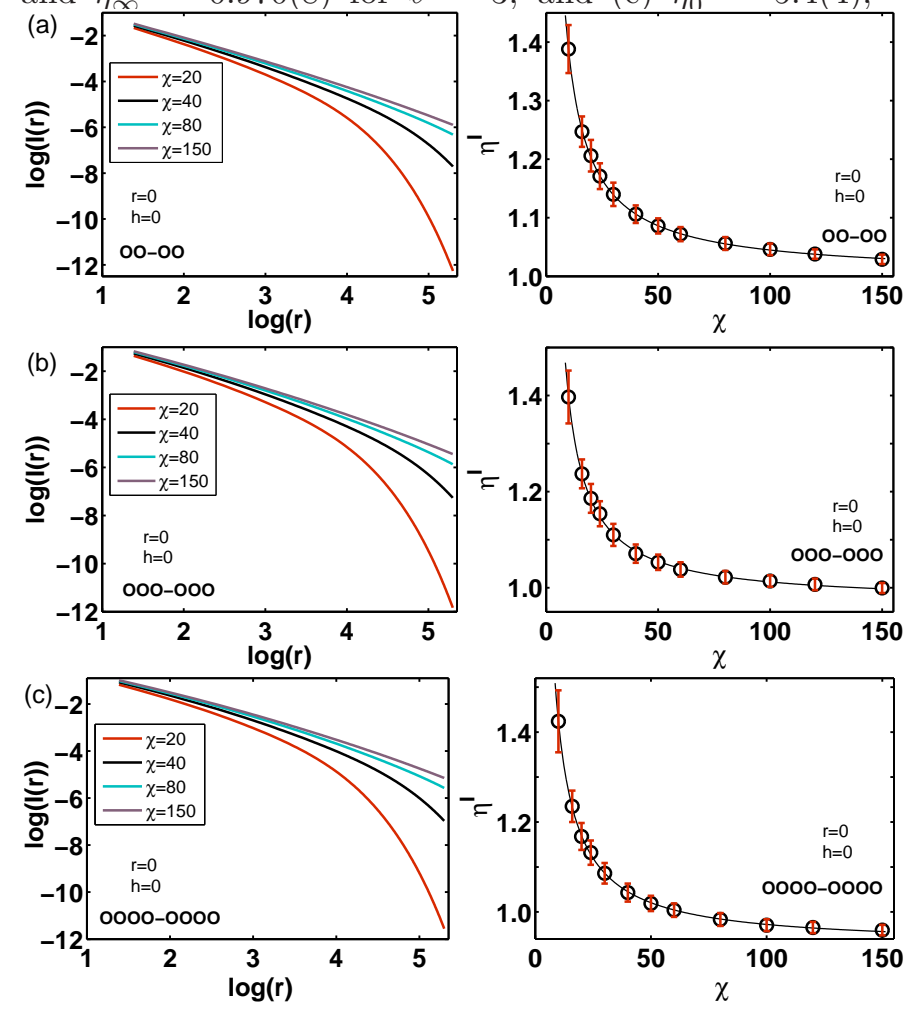

FIG. 10: (color online) Mutual information $I(r)$ as a function of the lattice distance $r=|i-j|$ for various truncation dimensions (left) and mutual information exponent $\eta^{I}(\chi)$ as a function of truncation dimension $\chi$ (right) with the block lengths $\ell_{A}=\ell_{B}=\ell$, i.e., (a) $\ell=2$, (b) $\ell=3$, and (c) $\ell=4$ for the transverse-field spin-1/2 XY model with $(\gamma, h)=(0.0,0.0)$. Mutual information exponent $\eta^{I}(\chi)$ (right) is extracted from the numerical fitting of the mutual information $I(\chi)$ (left) with the fitting function $\log I(r)=\eta^{I} \log r+a_{0}$ for the powerlaw decaying part.

$\alpha=-1.03(3)$ and $\eta_{\infty}^{I}=0.926(5)$ for $\ell=4$. For $(\gamma, h)=(0.0,0.5)$ in Fig. [1], the fitting constants are determined as (a) $\eta_{0}^{I}=3.3(2), \alpha=-0.92(3)$ and $\eta_{\infty}^{I}=1.008(4)$ for $\ell=2$, (b) $\eta_{0}^{I}=4.7(3), \alpha=-1.06(3)$ and $\eta_{\infty}^{I}=0.972(4)$ for $\ell=3$, and (c) $\eta_{0}^{I}=4.4(3)$, $\alpha=-0.99(4)$ and $\eta_{\infty}^{I}=0.922(6)$ for $\ell=4$. From these estimates, one may notice that for a given size of lattice-block $\ell$, critical exponents for $(\gamma, h)=(0.0,0.0)$ and $(\gamma, h)=(0.0,0.5)$ are very close values each other. However, the estimate values for $(\gamma, h)=(1.0,1.0)$ and $(\gamma, h)=(0.5,1.0)$ belonging to the Ising universality class are noticeably different from those for $(\gamma, h)=(0.0,0.0)$ and $(\gamma, h)=(0.0,0.5)$ belonging to the Gaussian universality class.
[1] S. Sachdev, Quantum Phase Transitions(Cambridge University, Cambridge,1999).
[2] P. M. Chaikin and T. C. Lubensky, Pinciples of Con- 

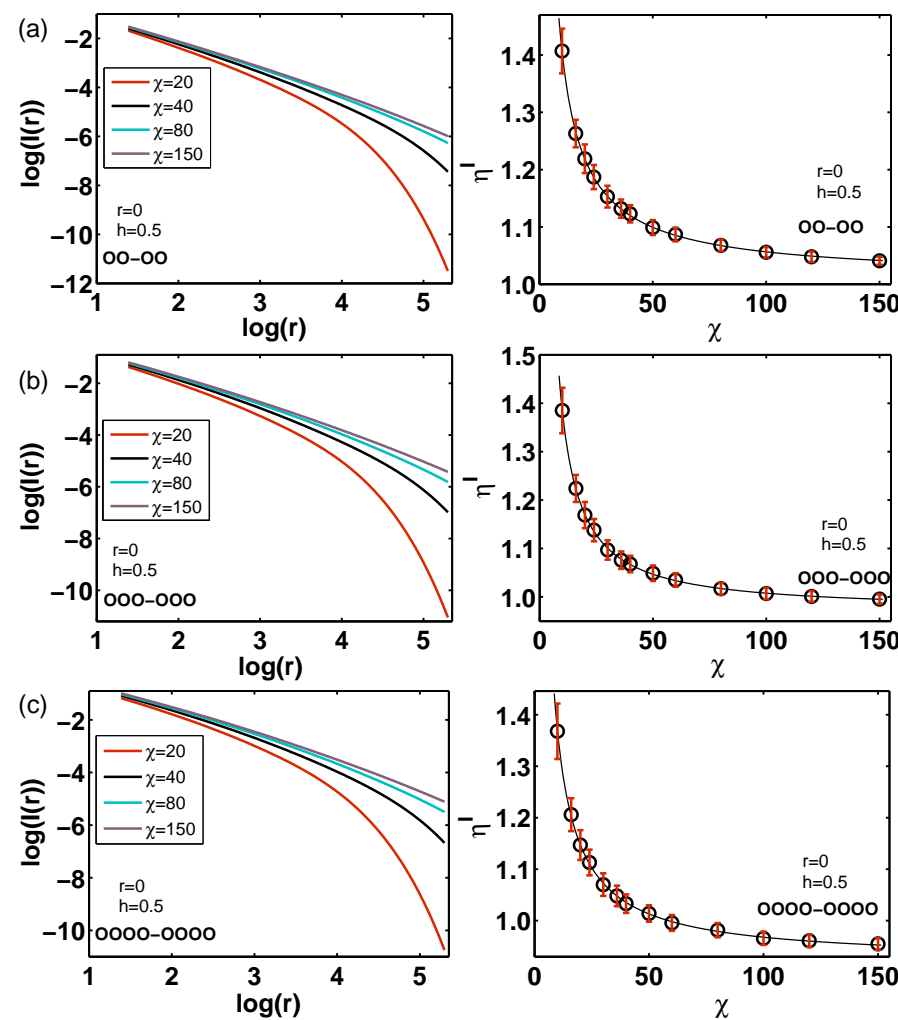

FIG. 11: (color online) Mutual information $I(r)$ as a function of the lattice distance $r=|i-j|$ for various truncation dimensions (left) and mutual information exponent $\eta^{I}(\chi)$ as a function of truncation dimension $\chi$ (right) with the block lengths $\ell_{A}=\ell_{B}=\ell$, i.e., (a) $\ell=2$, (b) $\ell=3$, and (c) $\ell=4$ for the transverse-field spin-1/2 XY model with $(\gamma, h)=(0.0,0.5)$. Mutual information exponent $\eta^{I}(\chi)$ (right) is extracted from the numerical fitting of the mutual information $I(\chi)$ (left) with the fitting function $\log I(r)=\eta^{I} \log r+a_{0}$ for the powerlaw decaying part.

densed Matter Physics (Cambridge University, Cambridge, (1995).

[3] L. Amico, R. Fazio, A. Osterloh, and V. Vedral, Rev. Mod.Phys. 80, 517 (2008); K. Modi, A. Brodutch, H. Cable, T. Paterek, and V. Vedral, ibid. 84, 1655 (2012).

[4] R. Dorner and V. Vedral, Int. J. Mod. Phys. B 27, 1345017 (2013).

[5] C. Adami and N. J. Cerf, Phys. Rev. A 56, 3470 (1997).

[6] B. Groisman, S. Popescu, and A. Winter, Phys. Rev. A 72, 032317 (2005).

[7] B. Schumacher and M. D. Westmoreland, Phys. Rev. A 74, 042305 (2006).

[8] V. Eisler, Z. Zimborás, Phys. Rev. B 89, 032321 (2014).

[9] G. Vidal, Phys. Rev. Lett. 91, 147902 (2003).

[10] G. Vidal, Phys. Rev. Lett. 98, 070201 (2007).

[11] A. Anfossi, P. Giorda, A. Montorsi, and F. Traversa, Phys. Rev. Lett. 95, 056402 (2005).

[12] M. M. Wolf, F. Verstraete, M. B. Hastings, and J. I. Cirac, Phys. Rev. Lett. 100, 070502 (2008).

[13] R. G. Melko, A. B. Kallin, and M. B. Hastings, Phys. Rev. B 82, 100409 (2010).

[14] Y.-X. Chen and S.-W. Li, Phys. Rev. A 81, 032120
(2010).

[15] R. R. P. Singh, M. B. Hastings, A. B. Kallin, and R. G. Melko, Phys. Rev. Lett. 106, 135701 (2011).

[16] J. Um, H. Park, and H. Hinrichsen, J. Stat. Mech. (2012) P10026.

[17] J. Wilms, J. Vidal, F. Verstraete, and S. Dusuel, J. Stat. Mech. (2012) P01023.

[18] Y. Huang and J. E. Moore, Phys. Rev. B 90, 220202 (2014).

[19] F. C. Alcaraz and M. A. Rajabpour, Phys. Rev. Lett. 111, 017201 (2013).

[20] J. M.Stéphan, J. Stat. Mech. (2014) P05010; J. M.Stéphan, Phys. Rev. B 90, 045424 (2014).

[21] F. C. Alcaraz and M. A. Rajabpour, Phys. Rev. B 90, 075132 (2014).

[22] F. C. Alcaraz and M. A. Rajabpour, Phys. Rev. B 91, 155122 (2015).

[23] M. Caraglio and F. Gliozzi, J. High Energy Phys. 11, 076 (2008).

[24] S. Furukawa, V. Pasquier, and J. Shiraishi, Phys. Rev. Lett. 102, 170602 (2009).

[25] P. Calabrese, J. Cardy, and E. Tonni, J. Stat. Mech. (2009) P11001.

[26] S. Marcovitch, A. Retzker, M.B. Plenio, and B. Reznik, Phys. Rev. A 80, 012325 (2009).

[27] H. Wichterich, J. Molina-Vilaplana, and S. Bose, Phys. Rev. A 80, 010304(R) (2009).

[28] H. Wichterich, J. Vidal, and S. Bose, Phys. Rev. A 81, 032311 (2010).

[29] H. Casini and M. Huerta, J. High Energy Phys. 03, 048 (2009).

[30] V. Alba, L. Tagliacozzo, and P. Calabrese, Phys. Rev. B 81, 060411(R) (2010).

[31] M. Fagotti and P. Calabrese, J. Stat. Mech. (2010) P04016.

[32] P. Calabrese, J. Cardy, and E. Tonni, J. Stat. Mech. (2011) P01021.

[33] R. A. Santos, V. Korepin, and S. Bose, Phys. Rev. A 84, 062307 (2011).

[34] P. Calabrese, J. Cardy, and E. Tonni, Phys. Rev. Lett. 109, 130502 (2012).

[35] M. Fagotti, Europhys. Lett. 97, 17007 (2012).

[36] P. Calabrese, J. Cardy, and E. Tonni, J. Stat. Mech. (2013) P02008.

[37] P. Calabrese, L. Tagliacozzo, and E. Tonni, J. Stat. Mech. (2013) P05002.

[38] A. Coser, L. Tagliacozzo, and E. Tonni, J. Stat.Mech. (2014) P01008.

[39] C. De Nobili, A. Coser, and E. Tonni, J. Stat.Mech. (2015) P01021.

[40] P. Ruggiero, E. Tonni, and P. Calabrese, J. Stat. Mech. (2018) 113101.

[41] S. S. Roy, S. N. Santalla, J. Rodríguez-Laguna, and G. Sierra, Phys. Rev. B 101, 195134 (2020).

[42] Y. H. Su, S. Y. Cho, B. Li, H. L. Wang, and H. Q. Zhou, J. Phys. Soc. Jpn. 81, 074003 (2012).

[43] J. Solyom and P. Pfeuty, Phys. Rev. B 24, 218 (1981).

[44] F. Y. Wu, Rev. Mod. Phys. 54, 235(1982).

[45] R. J. Baxter, Exactly Solved Models in Statistical Mechanics (Academic, London, 1982).

[46] P. P. Martin, Potts Models and Related Problems in Statistical Mechanics (World Scientific, Singapore, 1991).

[47] Y.-H. Su, B.-Q. Hu, S.-H. Li, and S. Y. Cho, Phys. Rev. E 88, 032110 (2013). 
[48] Y.-W. Dai, S. Y. Cho, M. T. Batchelor, and H.-Q. Zhou, Phys. Rev. E 89, 062142 (2014).

[49] C. Holzhey, F. Larsen, and F. Wilczek, Nucl. Phys. B424,443 (1994).

[50] G. Vidal, J. I. Latorre, E. Rico, and A. Kitaev, Phys. Rev. Lett. 90, 227902 (2003).

[51] J. I. Latorre, E. Rico, and G. Vidal, Quantum Inf. Comput. 4, 048 (2004).

[52] P. Calabrese and J. Cardy, J. Stat. Mech. (2004) P06002.

[53] B.-Q. Jin and V. E. Korepin, J. Stat. Phys. 116, 79 (2004).

[54] V. E. Korepin, Phys. Rev. Lett. 92, 096402 (2004)

[55] N. Laflorencie, E. S. Sørensen, M.-S. Chang, and I. Affleck, Phys. Rev. Lett. 96, 100603 (2006).

[56] S. Ryu and T. Takayanagi, Phys. Rev. Lett. 96, 181602 (2006).
[57] S. Ryu and T. Takayanagi, J. High Energy Phys. 08 (2006) 045.

[58] Y-W Dai, X-H Chen, S. Y. Cho, H-Q Zhou and D-X Yao, arXiv:1805.03464

[59] E. Lieb, T. Schultz, and D. Mattis, Ann. Phys. 16, 407 (1961).

[60] S. Katsura, Phys. Rev. 127, 1508 (1962); S. Katsura, ibid. 129, 2835 (1963).

[61] E. Lieb and D. Mattis, Mathematical Physics in One Dimension (Academic Press, New York and London,1966).

[62] P. Pfeuty, Ann. Phys. (NY). 57, 79 (1970).

[63] K. Damle and S. Sachdev, Phys. Rev. Lett. 76, 4412 (1996)

[64] J. E. Bunder and R. H. McKenzie, Phys. Rev. B 60, 344 (1999). 